\title{
From bedside to bench: regulation of host factors in SARS-CoV-2 infection
}

\author{
Samantha Y. Q. Ong ${ }^{1}$, Ilya M. Flyamer², Wendy A. Bickmore² and Simon C. Biddie (i)
}

\begin{abstract}
The zoonotic coronavirus SARS-CoV-2 (severe acute respiratory syndrome coronavirus-2), which causes COVID-19 (coronavirus disease-2019), has resulted in a pandemic. This has led to an urgent need to understand the molecular determinants of SARS-CoV-2 infection, factors associated with COVID-19 heterogeneity and severity, and therapeutic options for these patients. In this review, we discuss the role of host factors in SARS-CoV-2 infection and describe variations in host factor expression as mechanisms underlying the symptoms and severity of COVID-19. We focus on two host factors, angiotensin-converting enzyme 2 (ACE2) and transmembrane serine protease 2 (TMPRSS2), implicated in SARS-CoV-2 infection. We also discuss genetic variants associated with COVID-19 severity revealed in selected patients and based on genome-wide association studies (GWASs). Furthermore, we highlight important advances in cell and chromatin biology, such as single-cell RNA and chromatin sequencing and chromosomal conformation assays, as methods that may aid in the discovery of viral-host interactions in COVID-19. Understanding how regulation of host factor genes varies in physiological and pathological states might explain the heterogeneity observed in SARS-CoV-2 infection, help identify pathways for therapeutic development, and identify patients most likely to progress to severe COVID-19.
\end{abstract}

\section{Introduction}

The emergence of the novel coronavirus (CoV) severe acute respiratory syndrome (SARS) coronavirus 2 (SARS$\mathrm{CoV}-2)$ has resulted in a pandemic. In general, the heterogenous symptomatology following SARS-CoV-2 infection, which ranges from asymptomatic to severe life-threatening COVID-19, suggests the involvement of host factors. Epidemiological studies to date have implicated several host factors in severe COVID-19, including male sex, older age, cardiovascular disease, obesity, and diabetes $^{1,2}$. These host factors have behavioral, environmental, and genetic contributions ${ }^{3}$.

Sequencing of the SARS-CoV-2 genome has enabled comparative studies with other beta-coronaviruses to identify pathogenic features. For example, the structural similarity of the SARS-CoV-2 and SARS-CoV spike (S)

\footnotetext{
Correspondence: Simon C. Biddie (simon.biddie@ed.ac.uk)

${ }^{1}$ Intensive Care Medicine, NHS Lothian, Edinburgh, UK

${ }^{2} \mathrm{MRC}$ Human Genetics Unit, Institute of Genetics and Molecular Medicine, The

University of Edinburgh, Edinburgh EH4 2XU, UK
}

proteins has accelerated the identification of angiotensinconverting enzyme 2 (ACE2) as a key host factor for viral entry ${ }^{4}$. The endogenous role of ACE2 is to hydrolyse the vasoconstrictor peptide angiotensin. Attachment of SARS-CoV-2 to host cells is mediated by binding of the viral S1 subunit receptor-binding domain to ACE2, permitting entry and replication ${ }^{5}$. Following ACE2 engagement, proteolytic cleavage of $\mathrm{S} 2$ by host proteases, such as plasma membrane-associated type II transmembrane serine protease (TMPRSS2), mediates membrane fusion and cytosolic release of the viral RNA. TMPRSS2 is essential for the establishment of airway infection by SARS-CoV-2 and other zoonotic and human $\mathrm{CoVs}^{5}$.

In general, understanding host factor regulation through chromatin structure and DNA-binding factor analyses in various cell types and pathophysiological states is crucial. In this review, we discuss the transcriptional regulation of host factors associated with SARS-CoV-2 and severe COVID-19. We focus on key host factors for SARS-CoV-2 infection, namely, ACE2 and TMPRSS2, 
and examine their expression in cell types linked to symptoms. We also explore regulatory control and context-dependent changes in host factor expression associated with infection and risk phenotypes. Furthermore, we review genetic variants that might confer an increased risk of severe COVID-19 and outline methodologies to guide genotype-phenotype correlation for functional validation of host factors.

\section{ACE2 and TMPRSS2 tissue distribution and correlation with clinical presentation}

High-throughput sequencing of RNA (RNA-seq) has shown ubiquitous expression of ACE2 and TMPRSS2. $A C E 2$ expression is particularly enriched in the lungs, heart, kidneys, intestine, brain, skin, oral mucosa, and endothelial cells ${ }^{6}$ (Fig. 1a). TMPRSS2 is mainly expressed in the prostate, salivary glands, and luminal epithelia of the gastrointestinal, urogenital and respiratory tracts ${ }^{7}$ (Fig. 1b). Patterns of ACE2 expression correlate with susceptibility to infection with SARS-CoV ${ }^{8}$, leading to the hypothesis of higher SARS-CoV-2 tropism for tissues with higher $A C E 2$ expression and consequent symptomatology.

ACE2 and TMPRSS2 expression and pulmonary pathology

Mainly transmitted through droplets, aerosols, and direct contact, the predominant targets of SARS-CoV-2 are the upper respiratory epithelium and lungs. COVID19 typically presents with constitutional respiratory symptoms, with the majority of patients having pyrexia and dry cough ${ }^{9}$. The central role of the lung in COVID-19 is supported by a significant rise in SARS-CoV-2 viral load in these organs in more severe disease, which does not occur in stool or serum ${ }^{10}$. In $10-20 \%$ of patients, the disease progresses in severity with bilateral ground-glass opacities on chest computed tomography ${ }^{11}$. In the first wave of COVID-19, approximately $5-20 \%$ of patients became critically ill, requiring invasive mechanical ventilation due to acute respiratory distress syndrome (ARDS) with bilateral diffuse alveolar damage and cellular exudates on postmortem analysis ${ }^{12}$.

Given their role in SARS-CoV-2 infection, expression of the ACE2 and TMPRSS2 genes in the lung may contribute to the observed pathology. Immunohistochemistry demonstrates the highest expression of the ACE2 protein within the sinonasal cavity and pulmonary alveoli, which are presumptive sites of viral transmission and disease development ${ }^{13}$. Single-cell RNA-seq (scRNA-seq) of healthy adult lung tissue from eight donors showed that $<1 \%$ of cells in the lung parenchyma expressed ACE2 mRNA, $83 \%$ of which were surfactant-producing alveolar type 2 (AT2) cells ${ }^{14}$. A low proportion of $A C E 2^{+}$cells has been observed in other scRNA-seq datasets, with proportionally higher $\mathrm{ACE2}^{+} \mathrm{AT} 2$ cells than other cell types $^{13,15}$. Consistent with immunohistochemical evidence of ACE2 expression on apical surfaces ${ }^{13}$, this provides compelling evidence for that the virus targets AT2 cells. scRNA-seq studies have also found that a subset of $A C E 2^{+}$cells strongly coexpress TMPRSS 2 and other viral process-related genes, suggesting enhanced vulnerability of AT2 cells to SARS-CoV-2 ${ }^{13,16}$. AT2 cells may thus represent a potential viral reservoir for replication or alternatively undergo viral-induced cell death to initiate barrier damage and inflammation.

The proinflammatory state of COVID-19 may also contribute to ACE2 expression through proinflammatory transcription factor (TF) binding. scRNA-seq of the human lung revealed that $A C E 2$ expression is associated with expression of interferon-stimulated genes (ISGs) and components of the type I interferon (IFN) signaling pathway, which is central to the immediate antiviral response ${ }^{17}$. In primary human upper airway epithelial cells, stimulation with IFN- $\gamma$ and IFN- $\alpha 2$ leads to $A C E 2$ upregulation, possibly through signal transducer and activator of transcription-1 (STAT-1) binding sites in the ACE2 promoter $^{17}$. Similarly, human goblet secretory cells of the nasal epithelia exhibit ACE2 upregulation in response to influenza viruses, likely via an IFN pathway ${ }^{17}$. IFN-induced ACE2 expression may confer susceptibility to severe disease: local induction of IFN and ISG have been observed in the bronchoalveolar lavage (BAL) of critically ill patients ${ }^{18}$, and high circulating IFN levels have been shown to persist in later stages of severe COVID$19^{19}$. Nevertheless, IFN-induced expression has been shown to represent a non-functional truncated ACE2 isoform, suggesting that conditions of high IFN levels are unlikely to increase viral S-protein-mediated cellular entry to promote infection ${ }^{20}$. Furthermore, the paucity of IFN production and high viral loads observed in the incubation phase $\mathrm{e}^{21,22}$ suggest a protective role for IFN in early disease, which is currently being evaluated in clinical trials $^{23}$. The relationship between spatiotemporal kinetics and tissue specificity of IFN responses and host factor expression during SARS-COV-2 infection requires further study.

\section{Extrapulmonary involvement and host factor expression}

Several extrapulmonary manifestations of COVID-19 have been observed, from benign symptoms involving the eyes and gastrointestinal and nasal systems to severe organ failure involving the neurological, cardiac, and renal systems. Diarrhea occurs in approximately $5 \%$ of cases $^{24}$, while headache and anosmia have been described as common early symptoms ${ }^{25}$. Regarding severe cases, acute cardiac injury was seen in $12 \%$ of patients in a Chinese cohort $^{26}$. Severe acute kidney injury (AKI) requiring renal replacement therapy is common among critically ill patients, affecting $20-40 \%$ of those in the $\mathrm{ICU}^{1}$. 


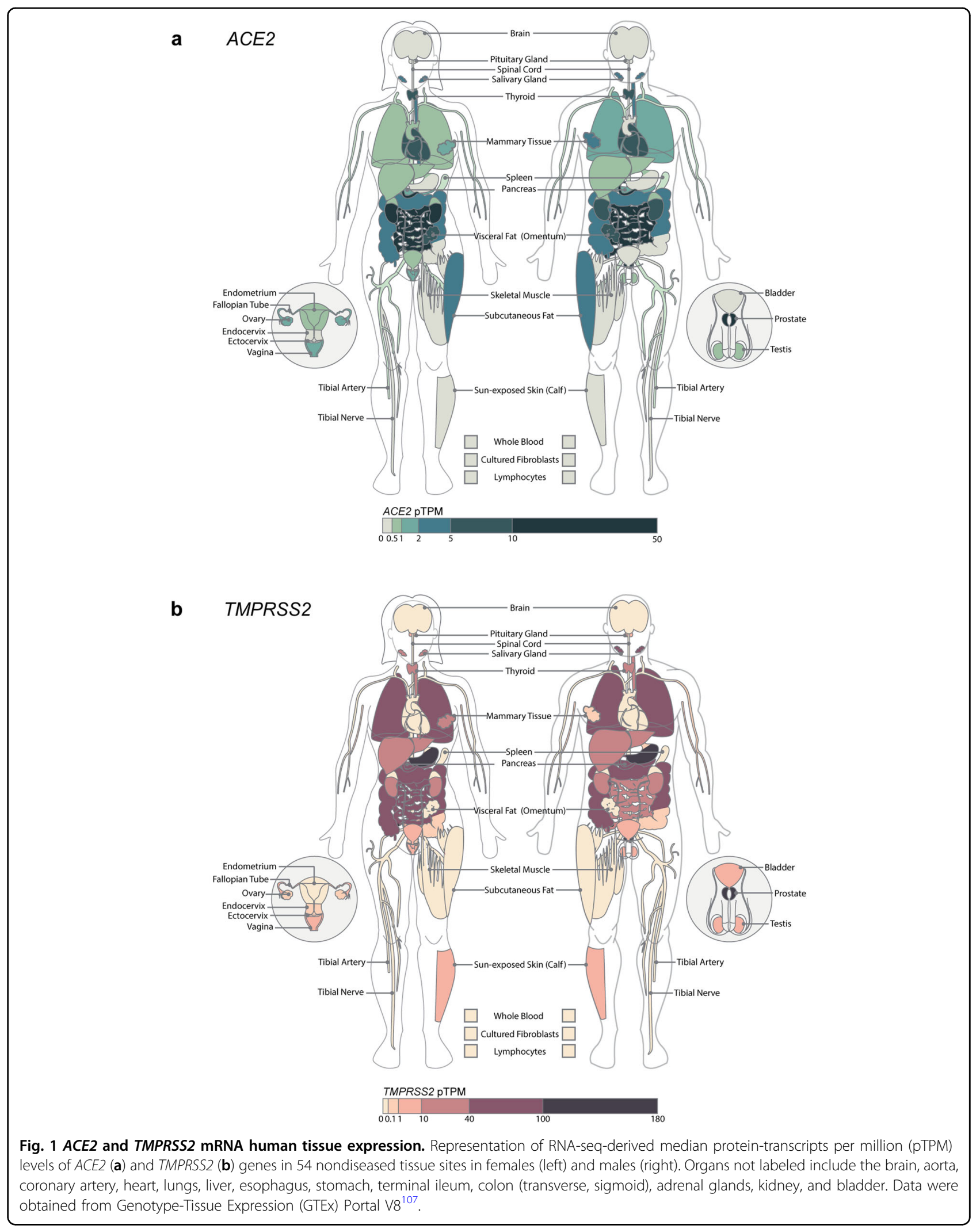


The severity and multisystem involvement observed in a subset of COVID-19 patients suggest a possible role for viral interaction with extrapulmonary host factors. Whole-tissue ACE2 mRNA expression and recent scRNA-seq studies reported high ACE2 expression in cardiac pericytes and cardiomyocytes, epithelial cells of the oral mucosa, ileum, stomach, colon and rectum, proximal renal tubules, and endothelial cells ${ }^{14,27,28}$. Similar to the lung, coexpression of ACE2 and TMPRSS2 in corneal, intestinal, and nasal epithelial cells is associated with activation of innate immune pathways, highlighting these cells as having potential roles in initiating viral infection ${ }^{29}$. TMPRSS2 expression facilitates SARSCoV-2 S protein-mediated entry in human $A C E 2^{+}$mature enterocytes in human small intestinal enteroids ${ }^{30}$. In the eye, high coexpression of ACE2 and TMPRSS2 has been observed in mouse cornea and human pterygium cell lines $^{31}$, which may explain SARS-CoV-2 detection in the tears and conjunctival secretions of COVID-19 patients with conjunctivitis ${ }^{32}$. Thus, organ systems expressing host entry factors can explain extrapulmonary symptoms such as diarrhea and conjunctivitis.

Given the greater risk of mortality in COVID-19 patients with cardiovascular disease, regulation of $A C E 2$ in the cardiovascular system is a mechanism that may be involved. For instance, upregulated $A C E 2$ expression has been observed in the myocardium of COVID-19 patients with heart failure ${ }^{33}$, and single-nucleus RNA-seq (snRNAseq) analysis has revealed higher $A C E 2$ expression in myocytes from patients with cardiomyopathy than in healthy controls. Under cardiac stress conditions, $A C E 2$ is repressed while $A C E$ is induced, with consequent increased angiotensin II-mediated cardiac hypertrophy and fibrosis ${ }^{34}$. This response to cardiac stress in endothelial cells is the result of forkhead box M1 (FOXM1) promoter binding. In addition, regulation of $A C E 2$ in the cardiovascular system appears to be cell-specific, as $A C E 2$ was found to be expressed at low levels in cardiac fibroblasts but highly expressed in myocytes, macrophages and endothelial, and smooth muscle cells ${ }^{35}$.

Postmortem studies have provided direct evidence of viral RNA in the lungs and pharynx and, to a lesser extent, in the renal tubules, liver, heart, brain, and blood ${ }^{36}$. Similarly, endothelial dysfunction in postmortem lung and kidney tissue is associated with viral inclusion bodies, suggesting direct viral infection ${ }^{37}$. Hence, differential tissue expression of ACE2 and TMPRSS2 might link viral injury to organ systems and may explain the observed correlation between high respiratory and plasma viral loads and severe COVID-19 and mortality ${ }^{38,39}$. Other studies, however, have reported no association between viral load and disease severity ${ }^{40}$. This is supported by a systematic meta-analysis of viral load dynamics in COVID-19 whereby no viable virus was detected beyond day nine of the disease, despite prolonged SARS-CoV-2 RNA shedding in respiratory and stool samples ${ }^{10}$. Furthermore, a postmortem study demonstrated disconnect between SARS-CoV-2 detection and organ inflammation and dysfunction ${ }^{41}$. Together, these results suggest that disease progression and mortality may not be directly virus dependent but rather driven by aberrant host immune responses.

\section{Host factor expression associated with COVID-19 risk factors}

Male sex has been independently associated with severe COVID-19 disease at presentation, the need for intensive care unit admission, and higher case fatality rates ${ }^{42}$. Differential host factor expression may explain the sex difference in COVID-19 (Fig. 1a, b), though the evidence is conflicting. A recent meta-analysis of scRNA-seq studies showed increased overall ACE2 and TMPRSS2 lung expression in males ${ }^{43}$; in contrast, others have found no significant sex difference with regard to $A C E 2$ expres$\operatorname{sion}^{44,45}$. Given the relatively low expression of $A C E 2$ and predominant AT2 cell expression, data for whole tissues compared to single-cell studies may also mask subtle differences. It is also noteworthy that while $A C E 2$ is located on the X chromosome, analysis of GTex data suggests that $A C E 2$ escapes $\mathrm{X}$ chromosome inactivation, resulting in sex-biased gene expression ${ }^{46}$. Sex differences in $A C E 2$ expression may also be due to regulation by hormone-dependent TFs. In female mice, ACE2 basal activity in the kidney was repressed by estrogen, suggesting estrogen receptor (ER) response elements at the $A C E 2$ locus $^{47}$. In the elderly human male atrial myocardium, ex vivo administration of estrogen induced $A C E 2$ mRNA through an ER $\alpha$-dependent mechanism ${ }^{48}$. Although these findings support $A C E 2$ control via an ER response element, differences in the response might vary by tissue type, sex, age, and pathological state.

DNase-seq has revealed differential shared and tissuespecific regulatory element activation for the ACE2 and TMPRSS2 $\operatorname{loci}^{49}$ (Fig. 2a), likely regulated by cell-specific $\mathrm{TF}$ binding at regulatory elements ${ }^{50}$. ENCODE datasets show binding of the insulator CCCTC-binding factor (CTCF) at shared sites for ACE2 and TMPRSS2, whereas activator protein-1 (AP-1) subunits bind to the tissuespecific $5^{\prime}$ upstream element of $A C E 2$, suggesting that variable host factor gene expression may be explained by differential engagement of regulatory elements and selective TF binding.

Regulation of TMPRSS2 has primarily been explored in prostate cancer. In this context, TMPRSS 2 is induced by androgens through a distal androgen receptor (AR) binding enhancer ${ }^{51}$, resulting in AR-induced expression of a TMPRSS2-ERG (ETS-related gene) fusion gene conferring prostate cancer pathogenicity ${ }^{52}$. Mapping of an AR 


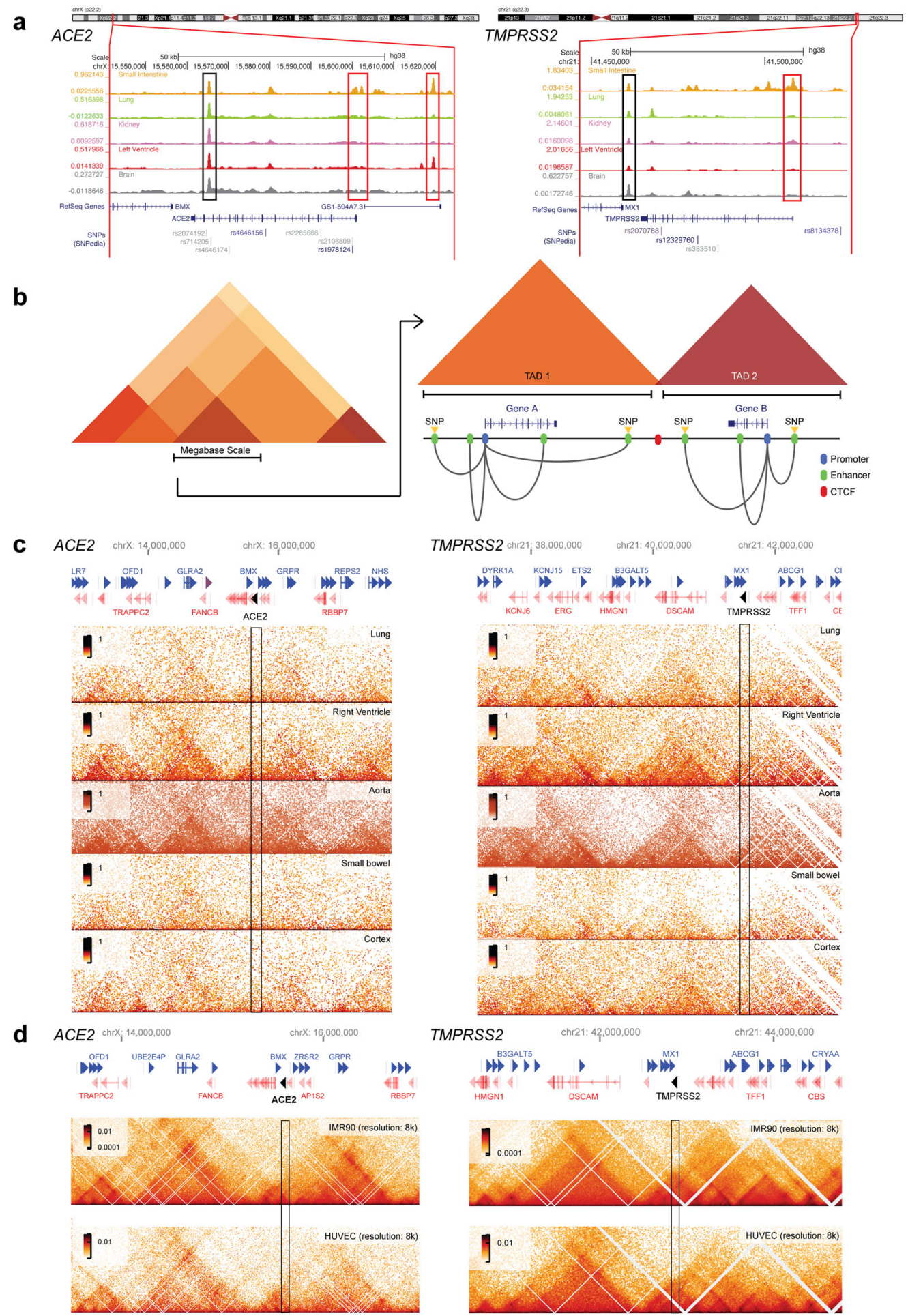

Fig. 2 (See legend on next page.) 
(see figure on previous page)

Fig. 2 ACE2 and TMPRSS2 regulatory elements and tissue-invariant topologically-associated domains. a Human tissue DNase-seq signals for ACE2 [left] and TMPRSS2 [right] loci. Tissue-specific regulatory elements (red bar) and shared regulatory elements (black bar) are highlighted. Datasets were obtained from the Encyclopedia of DNA Elements (ENCODE) 2018 data release for $\mathrm{Hg}_{3} 8^{49}$ and visualized via the UCSC genome browser. Normalized signal replicate pools are depicted for each tissue type. ENCODE experimental designations are ENCSR747YZZ (small intestine), ENCSR619JTC (lung), ENCSR955NXV (kidney), ENCSR871OSL (left ventricle) and ENCSR341MVE (brain). b Schematic representation of topologicallyassociated domains (TADs) showing boundaries for regulatory elements that control expression of the genes within a TAD. The formation of a TAD is dependent on insulator elements bound by CCCTC-binding factor (CTCF). Enhancer association with promoters via long-range interactions may act to regulate expression. Some enhancers harbor SNPs that might alter the expression pattern of genes within a TAD. $\mathbf{c}$ Hi-C data for human tissues depict chromosome conformation into TADs around ACE2 (left) and TMPRSS2 (right). The locations of the respective genes are indicated in black. Lung, right ventricle, small bowel, and cortex datasets were obtained from Schmitt et al. ${ }^{94}$. Aorta data were obtained from Leung et al. ${ }^{108}$. Hi-C datasets were visualized in HiGlass ${ }^{109}$ at 20-kb resolution. Datasets are aligned to human genome build $\mathrm{Hg} 38$. d Hi-C data for human lung fibroblast (IMR-90) and human endothelial (HUVEC) cell lines show higher-order chromosome organization as TADs. The genome locations of ACE2 (left) and TMPRSS2 (right) are indicated in black. Datasets aligned to human genome build $\mathrm{Hg} 18$ were obtained from Rao et al. ${ }^{95}$ and visualized in $\mathrm{HiGlass}{ }^{109}$ at $8-k b$ resolution.

response element to the induction of TMPRSS2 expression has led to the hypothesis that androgenic phenotypes are risk factors for COVID-19 ${ }^{53}$. Epidemiological studies have also suggested androgenic alopecia as a separate risk profile for COVID- $19^{54}$. However, AR blockade in mice did not alter lung TMPRSS2 expression ${ }^{55}$, suggesting that lung TMPRSS2 may not be androgen regulated, possibly through tissue-specific enhancer inaccessibility. As TMPRSS 2 promoters in mammary epithelial cells have been found to be responsive to estrogens ${ }^{56}$, the role of TMPRSS2 in conferring risk in men through sex-specific expression in relevant tissues remains unclear.

Moreover, sex-biased behavior may contribute to male predominance of COVID-19. Higher rates of smoking among men have been hypothesized to contribute to male predisposition towards the disease ${ }^{57}$, and active smoking is implicated as a negative prognostic indicator for COVID $-19^{24}$. This would be consistent with increased $A C E 2$ in the bronchial epithelium ${ }^{58}$ and small airway epithelium of chronic smokers ${ }^{45,59}$. scRNA-seq data showed dose-dependent upregulation of $A C E 2$ by cigarette smoke in rodent and human lungs, with chronic smoke exposure triggering an expansion of $A C E 2^{+}$ mucus-secreting goblet cells ${ }^{60}$.

Age-related dynamics in host factor expression may similarly explain the greater severity and mortality that occurs among older patents ${ }^{9,61}$. Indeed, older age is associated with higher viral load in oropharyngeal salivary and respiratory samples ${ }^{62}$. Lung $A C E 2$ expression has been shown to increase $\sim 1.2$-fold with every 10-year increase in age $^{44}$. scRNA-seq of developing mouse lungs and temporally resolved RNA-in situ hybridization (ISH) analysis also found an age-related increase in TMPRSS2 expression $^{63}$. Single-nucleus ATAC-seq (snATAC-seq) in human AT2 cells demonstrated age-associated changes in accessible chromatin around TMPRSS2 at sites harboring sequence motifs for multiple TFs, including forkhead box A (FOXA), C/EBP $\alpha$, the proinflammatory factors STAT, IFN-regulatory factor (IRF), and AP-1, suggesting age- dependent inflammatory regulation of TMPRSS2 ${ }^{64}$. High ACE2 and TMPRSS2 levels in the elderly may therefore be associated with increased viral infection, although altered immune response and physiology may also explain divergence in severity with age extremes.

Although lower nasal epithelial ACE2 expression reported in children ${ }^{65}$ has been suggested to explain the milder disease observed in this population ${ }^{66}$, others have found that pulmonary ACE2 is not reduced in children ${ }^{13}$. Furthermore, scRNA-seq and immunohistochemistry of human respiratory tract tissue indicated no significant difference in ACE2 localization in those with independent risk factors for severe COVID-19, such as male sex, asthma, cardiovascular disease, COPD, diabetes, and smoking, compared to age-matched healthy controls ${ }^{1,2,13}$. This contradictory evidence suggests a complex relationship between pulmonary ACE2 expression and COVID-19 as both a viral receptor and protective factor, implicating other host factors in regulating disease severity and susceptibility.

\section{Host genetic variants and the spectrum of COVID- 19}

Host genetic factors have been implicated in influenza and zoonotic diseases such as SARS and MERS ${ }^{67,68}$. Genetic variants may alter disease through viral infection, replication or immune function by altering host gene expression or protein function, leading to heterogeneous severity.

\section{Genetic variants of ACE2 and TMPRSS2}

Human variants of $A C E 2$ could alter the protein interface with the SARS-CoV-2 S protein. Moreover, as ACE2 is located on the $\mathrm{X}$ chromosome, hemizygosity for harmful variants might contribute to poor outcomes in men with COVID-19. Genomic variant analysis from Genome Aggregation Database (gnomAD) ${ }^{69}$ reveals a very low frequency of non-synonymous $A C E 2$ variants, including those predicted to be loss-of-function (Fig. 3). 


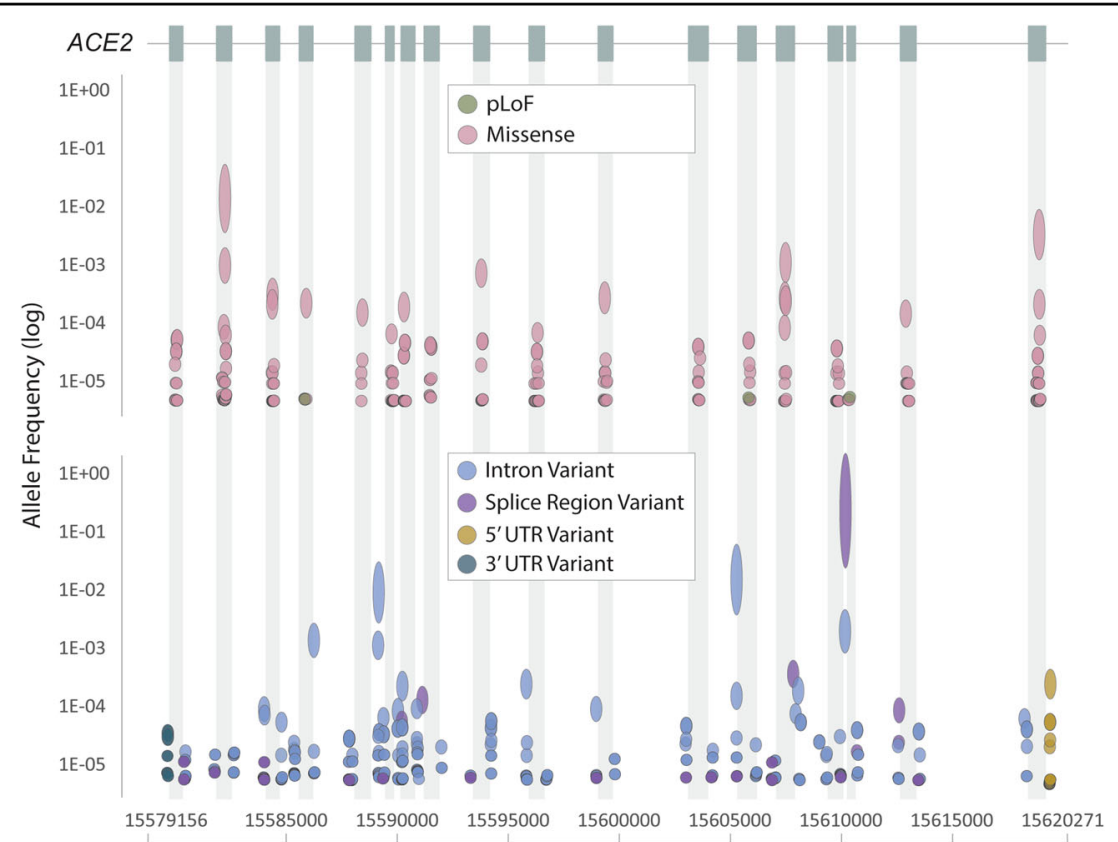

Fig. 3 Single-nucleotide polymorphism (SNP) allelic frequencies of angiotensin-converting enzyme 2 (ACE2). ACE2 (chromosome X: 15579156-15620271) exon coding transcripts are represented by boxes and corresponding shaded areas. Predicted loss-of-function (pLOF) and missense variants (top panel) and intron, splice region, 5' untranslated region (UTR), and 3'UTR variants (bottom panel) are shown. The size of the dot reflects the minor allele count (range: 1-45,478), with minor allele frequency represented on the $y$-axis. Data were obtained from gnomAD v2.1.1 ${ }^{69}$.

Furthermore, no correlation between rare deleterious $A C E 2$ exonic and splice junction variants with COVID-19 was observed in an Italian population ${ }^{70}$.

Given the role of ACE2 in cardiovascular disease and lung disease, including ARDS, ACE2 polymorphisms are of interest beyond COVID-19. For example, a common exonic SNP in $A C E 2$, rs2285666 (Fig. 2a), has been implicated in the risk of cardiovascular disease and type 2 diabetes mellitus, with the rare A allele found at a frequency of $0.2 \%$ in European populations and $0.55 \%$ in East Asians $^{70}$. Two ACE2 intronic SNPs, rs2074192 and rs2106809, associated with left ventricular hypertension are expression quantitative trait loci (eQTLs) for $A C E 2$ and may therefore link $A C E 2$ cardiac expression with high penetrance of alleles, increasing $A C E 2$ expression in East Asians ${ }^{61}$. ACE2 variants could therefore be linked to risk factors for COVID-19, but further clarification within comorbid subgroups is required.

For TMPRSS2, the frequency of rare deleterious and common exonic variants speculated to alter protein levels or activity varies between populations. For common variants, two haplotypes have been identified. One is linked to an eQTL harboring rs8134378 and mapping to an AR regulatory element $15 \mathrm{~kb}$ upstream of the TMPRSS2 CpG island $^{70}$. The second includes the intronic SNP rs2070788 (Fig. 2a), which has been found in small-scale genomewide association studies (GWASs) to be linked to an increased risk of influenza ${ }^{71}$. Less is known about
TMPRSS2 variants, warranting further investigation. However, in the first two published GWASs for severe COVID-19, there was no significant association with $A C E 2$ or TMPRSS2 variants, suggesting that variants of these factors do not contribute to COVID-19 severity ${ }^{3,72}$.

\section{Genetic variants in intracellular RNA sensing and type I interferon signaling}

Based upon the gene mutations implicated in lifethreatening influenza pneumonia, rare but predicted deleterious mutations have been examined in patients with severe COVID- $19^{72}$. Mutations in the genes encoding double-stranded RNA sensor TLR3 and in downstream components of type I IFN cell-intrinsic immunity have been detected, including UNC93B1, which forms a complex with and is involved in intracellular trafficking of $\mathrm{TLR}^{73}$ and other downstream components, such as TRIF and TBK1, a key kinase linking pattern recognition receptor activation and activation of ISGs. Mutations in the type I IFN amplification pathway were also found, including in the genes coding for IRF7 and the cell surface receptors IFNAR1 and IFNAR2. In addition, common variants at IFNAR2 were found in a GWAS for severe COVID- $19^{3}$. Although no rare variants in the JAK/STAT pathway downstream of IFNAR1/2 have been reported in severe COVID-19, variants at TYK2 on chromosome 19 were reported in the GWAS of a UK intensive care cohort of COVID-19 patients with critical respiratory failure ${ }^{3}$. Of 


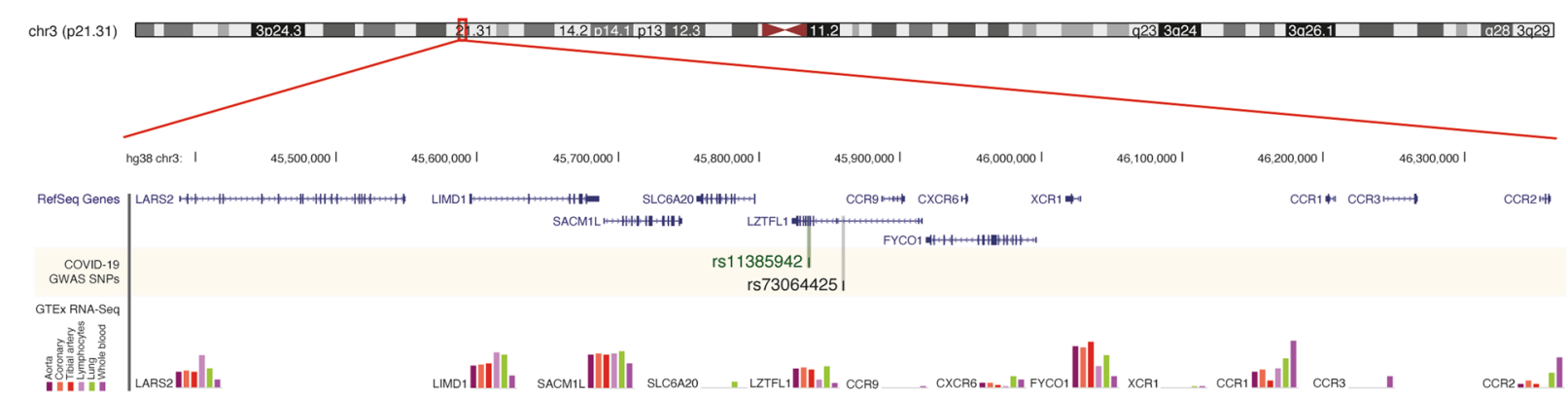

Fig. 4 The 3p21.31 locus harbors significant single-nucleotide variants from the COVID-19 GWAS. The COVID-19 GWAS identified SNPS mapping to the 3p21.31 locus. The lead SNP from each study (rs11385942 from Ellinghaus et al. ${ }^{82}$; rs73064425 from Pairo-Castineira et al. ${ }^{3}$ ) mapped to LZTFL1. Images were generated from the UCSC genome browser (hg38, chr3:45,388,000-46,361,000), showing RefSeq genes and Genotype-Tissue Expression (GTEx) RNA-seq data for selected tissues ${ }^{107}$.

note, patients with complete TYK2 deficiency are susceptible to bacterial and viral infection ${ }^{74}$. The contribution of genetic variants to reduced IFN signaling in severe COVID-19 is further supported by observations of impaired IFN responsiveness in neutrophils from patients with severe COVID-19 $9^{75}$. Nonetheless, the WHO Solidarity Trial has shown that treatment of COVID-19 patients with IFN- $\beta 1$ a did not decrease mortality or the need for mechanical ventilation ${ }^{76}$.

Some critical COVID-19 patients with mutations in IFN pathways exhibit reduced serum IFN- $\alpha^{72}$, though other studies have reported higher IFN- $\gamma$ in helper T cells of patients who died from COVID-1977. IFN- $\gamma$ is linked to IL-6 signaling through the IL-6 receptor (IL6R) ${ }^{78}$, suggesting interplay between IFN and interleukin signaling in acute inflammation. Genetic variants of IL6R are associated with a lower risk of hospitalization following SARS$\mathrm{CoV}-2$ infection $^{79}$, yet these variants were not significant in the COVID-19 GWASs above. Nevertheless, modulation of the IL6R axis using inhibitors, such as tocilizumab and sarilumab, was shown in the REMAP-CAP and EMPACTA trials to reduce the need for mechanical ventilation and reduce mortality in patients with severe COVID-19 ${ }^{80,81}$.

In the first published GWAS for severe COVID-19, a significant cross-replicating locus at 3p21.31 was found in Italian and Spanish populations ${ }^{82}$, spanning six genes: LC6A20, LZTFL1, CCR9, FYCO1, CXCR6, and XCR1 (Fig. 4). The insertion-deletion GA risk variant of rs11385942 in an intron of LZTFL1 is associated with reduced CXCR6 but increased SLCA2O expression. Interestingly, the risk allele was detected frequently in patients who required mechanical ventilation, and furthermore, the few individuals homozygous for the risk allele were younger, suggesting an association with COVID-19 severity. The frequency of risk allele heterozygosity varies between populations, occurring in less than $0.5 \%$ in China and $37.8 \%$ in Bangladesh ${ }^{82}$. Moreover, a GWAS of the UK intensive care cohort of COVID-19 patients with critical respiratory failure ${ }^{3}$ replicated the significance of the chromosome 3 locus.

The genes at the $3 \mathrm{p} 21.31$ locus have plausible roles in COVID-19 and lung injury. SLC6A20 encodes a sodiumdependent amino transporter that has been shown to interact with ACE2 in a Xenopus oocyte expression system $^{83}$. CXCR6 is a marker of lung-resident memory $\mathrm{CD}^{+}$ $\mathrm{T}$ cells, producing cytokines in response to stimuli ${ }^{84 .}$ Its ligand, CXCL16, is elevated in the serum of patients with acute lung injury, and both ligand CXCL16 and CXCR6 receptor genes are upregulated in models of lung injury ${ }^{9}$. CXCL16-CXCR6 acts to disrupt epithelial integrity through downregulation of E-cadherin in lung injury', which might represent the fibrosis observed in ARDS. CCR9, as part of a CCL25-CCR9 chemokine axis, has been shown to mediate gut-specific recruitment of lung IFN- $\gamma$-producing $\mathrm{CCR}^{+} \mathrm{CD}^{+}{ }^{+} \mathrm{T}$-cells in influenza, contributing to the gut injury observed in viral infection ${ }^{64}$. FYCO1, encoding an autophagy adaptor protein, was found to be upregulated in intensive care patients with sepsis $^{85}$.

The second locus uncovered by Ellinghaus et al. maps to the $A B O$ blood group gene, whereby group A confers a higher risk of severe COVID-19 and group O demonstrates a protective effect ${ }^{82}$. The plausibility of an association is supported by an epidemiological study in a Chinese population, showing increased COVID-19 risk with blood group A and reduced risk with blood group $\mathrm{O}^{86}$. However, the association of $\mathrm{ABO}$ blood groups with COVID-19 severity was not replicated by the GenOMICC study $^{3}$. Additional loci associated with COVID-associated ICU admission include the $O A S$ gene cluster on chromosome 12 and DPP9 on chromosome $19^{3}$. OAS genes are activated by IFN and are involved in double-stranded RNA degradation pathways in the early innate antiviral response $^{87,88}$. DPP9 encodes a serine protease implicated in lung fibrosis ${ }^{89}$. These pathways may therefore represent 
therapeutic targets to mitigate progression to critical respiratory failure in COVID-19.

\section{Mechanisms of genetic variants driving increased disease risk}

Most GWAS disease-risk loci are in noncoding regions of the genome ${ }^{90}$. The cataloging of human regulatory elements on a genome-scale across multiple cell and tissue types has uncovered a correlation between accessible chromatin, DNA-binding factors and $\mathrm{SNPs}^{91}$. These large-scale studies implicate altered TF binding as a core feature of noncoding genetic variants. For example, in lymphoblastoid cell lines, interindividual variation in NF$\mathrm{kB}$ binding has been associated with genetic variants and alterations in gene expression ${ }^{92}$. Genetic variation could, therefore, alter responses to stimuli, such as infection, between individuals and thus influence severity.

To infer phenotypic consequences of genetic variants, functional assignment of genetic variants to gene regulation is required but remains challenging. Chromatin structure can be used to aid functional characterization of variants. Chromatin higher-order structures have been found to form topologically-associated domains (TADs), regions of chromatin that show high interaction frequencies separated by boundary regions ${ }^{93}$ (Fig. 2b). At the megabase scale, TADs are generally stable between cell types and tissues ${ }^{94,95}$. TAD boundaries are marked by binding sites for CTCF, which impedes cohesin-driven loop extrusion, the process responsible for TAD formation. For the most part, enhancers are generally found in the same TAD as the gene(s) they regulate ${ }^{96}$. Therefore, incorporating information on chromatin structure, including smaller scale accessibility information with larger scale TADs, may aid in the discovery of associations between noncoding variants and genes to guide finemapping experiments ${ }^{97}$ and has allowed functional refinement of SNPs and eQTLs ${ }^{98}$.

ACE2 and TMPRSS2 are located within tissueconserved TADs (Fig. 2c, d). However, eQTL analysis of SNPs at the $A C E 2$ locus did not reveal an association with gene expression in lung tissue ${ }^{99}$. Within the $A C E 2$ TAD (hg38 chrX:15,300,000-15,600,000), long-distance interactions were observed between multiple SNPs and other genes associated with eQTLs in the lung, such as PIR, a modulator of NF-kB signaling, and $C A 5 B$, which is involved in acid-base metabolism ${ }^{99}$.

Incorporating open chromatin might also contribute to the identification of functional genetic variants. By applying snATAC-seq to healthy human lungs, agedependent accessible regions in TMPRSS2-expressing AT2 cells were found to be enriched for genes associated with inflammatory signals ${ }^{100}$. In AT2 cells, age-dependent accessible chromatin near TMPRSS2 correlated with sequence variants, including common variants with a minor allele frequency $>1 \%^{100}$. These variants are predicted to disrupt sequence motifs of DNA-binding factors and thus alter expression and are associated with GWAS phenotypes for emphysema, asthma, and bacterial pneumonia $^{100}$.

\section{Conclusion}

The sequalae following SARS-CoV-2 infection are highly heterogeneous, from asymptomatic to multiorgan failure, and host factors are likely variables involved in COVID-19 severity ${ }^{101}$. Risk factors for COVID-19 contribute to modified disease risk through changes in viral-host interactions, most likely through host factor expression. ACE2 and TMPRSS2 have been identified as SARS-CoV-2 tropic factors. However, ACE2 was found to be protective against pulmonary injury through maintenance of vascular competence in mouse models of acute lung injury ${ }^{102}$. The balance between viral exploitation of host factors and the role of that factor in COVID-19 pathophysiology is not clear. Furthermore, ACE2 and TMPRSS2 genetic variants are not convincingly associated with COVID-19 severity ${ }^{3,82}$. Other important host factors involved in viral-host interactions, viral protein processing, and immune function are more likely to contribute to severity. The therapeutic effect of glucocorticoids, such as dexamethasone ${ }^{103}$ or hydrocortisone ${ }^{104}$, in decreasing COVID-19 mortality or the need for organ support strongly suggests an inflammatory process in severe disease. IFN pathways are also likely to play key roles in COVID-19, and their impairment might explain progression to critical illness. While the WHO Solidarity trial does not support the use of IFN as a treatment modality ${ }^{76}$, questions on the timing and regime remain while highlighting a need for alternate targets. Exploring the expression variation and regulatory control of other host factors, such as the 3p21.31 locus identified from COVID19 GWASs, will aid in our understanding of the disease spectrum and uncover therapeutic targets ${ }^{3,82}$. It will also be important to integrate comorbidities with the genetic variants implicated in COVID-19 to understand the contributions of risk factors to COVID-19 severity.

The identification of functional regulatory elements in health and pathological states is a crucial pursuit, with many approaches being developed following the mapping of noncoding genomic regions. We have highlighted a number of methods that have been employed to identify regulatory elements, but their use in conglomerate is likely required, taking a multiomics approach to identify functional consequences. This is particularly important for understanding dynamic changes in regulatory control, for instance, during inflammation. The development of single-cell methodologies, as an example, has also revolutionised our discovery and understanding of cell types and heterogeneous RNA expression in complex tissues. 
Nevertheless, functional studies of complex tissues have been largely limited to model organisms, with questionable translational potential. The development of human organoid systems may allow for a more relevant understanding of the molecular processes that drive variation in disease presentation and progression. Combining these approaches with functional assays for regulatory control of gene expression by using, for example, high-throughput CRISPR-based perturbation methods ${ }^{105}$ or a highthroughput self-expressing reporter system to identify functional enhancer elements in cell-specific control ${ }^{106}$, would allow dissection of the roles of regulatory elements, genetic variants or biological pathways in disease processes such as viral infection. Such approaches will allow the identification of variables to allow risk stratification for public health initiatives, resource allocation for at-risk groups and the development of novel therapeutics.

\section{Acknowledgements}

The views expressed are those of the authors and independent from the NHS. S.C.B. is supported by the Scottish Clinical Research Excellence Development Scheme (SCREDS) and the NHS Education for Scotland (NES)/Chief Science Office (CSO), Scotland, UK. I.M.F. and W.A.B. are supported by MRC University Unit grant (MC_UU_00007/2).

\section{Conflict of interest}

The authors declare no competing interests.

\section{Publisher's note}

Springer Nature remains neutral with regard to jurisdictional claims in published maps and institutional affiliations.

Received: 30 November 2020 Revised: 1 February 2021 Accepted: 15 February 2021.

Published online: 7 April 2021

\section{References}

1. Cummings, M. J. et al. Epidemiology, clinical course, and outcomes of critically ill adults with COVID-19 in New York City: a prospective cohort study. Lancet 395, 1763-1770 (2020).

2. Grasselli, G. et al. Baseline characteristics and outcomes of 1591 patients infected with SARS-CoV-2 admitted to ICUs of the Lombardy Region, Italy. JAMA 323, 1574-1581 (2020).

3. Pairo-Castineira, E. et al. Genetic mechanisms of critical illness in Covid-19. Nature https://doi.org/10.1038/s41586-020-03065-y (2020).

4. Wan, Y., Shang, J., Graham, R., Baric, R. S. \& Li, F. Receptor recognition by the novel coronavirus from Wuhan: an analysis based on decade-long structural studies of SARS coronavirus. J. Virol. 94, e00127-20 (2020).

5. Hoffmann, M., Kleine-Weber, H. \& Pöhlmann, S. A multibasic cleavage site in the spike protein of SARS-CoV-2 is essential for infection of human lung cells. Mol. Cell 78, 779-784. e5 (2020).

6. Li, M. Y., Li, L., Zhang, Y. \& Wang, X. S. Expression of the SARS-CoV-2 cell receptor gene ACE2 in a wide variety of human tissues. Infect. Dis. Poverty $\mathbf{9}$, 45 (2020).

7. Vaarala, M. H., Porvari, K., Kyllönen, A., Lukkarinen, O. \& Vihko, P. The TMPRSS2 gene encoding transmembrane serine protease is overexpressed in a majority of prostate cancer patients: detection of mutated TMPRSS2 form in a case of aggressive disease. Int. J. Cancer 94, 705-710 (2001).

8. Yang, X. H. et al. Mice transgenic for human angiotensin-converting enzyme 2 provide a model for SARS coronavirus infection. Comp. Med. 57, 450-459 (2007).
9. Zhou, F. et al. Clinical course and risk factors for mortality of adult inpatients with COVID-19 in Wuhan, China: a retrospective cohort study. Lancet 295, 1054-1062 (2020).

10. Zheng, S. et al. Viral load dynamics and disease severity in patients infected with SARS-CoV-2 in Zhejiang province, China, January-March 2020: Retrospective cohort study. BMJ 369, m1443 (2020).

11. Pan, F. et al. Time course of lung changes on chest $C T$ during recovery From 2019 novel coronavirus (COVID-19) pneumonia. Radiology 295, 715-721 (2020).

12. $\mathrm{Xu}, \mathrm{Z}$. et al. Pathological findings of COVID-19 associated with acute respiratory distress syndrome. Lancet Respir. Med. 8, 420-422 (2020).

13. Ortiz, M. E. et al. Heterogeneous expression of the SARS-Coronavirus-2 receptor ACE2 in the human respiratory tract. EBioMedicine 60, 102976 (2020).

14. Zhao, $Y$. et al. Single-cell RNA expression profiling of ACE2, the receptor of SARS-CoV-2. Am. J. Respir. Crit. Care Med. 202, 756-759 (2020).

15. Travaglini, K. J. et al. A molecular cell atlas of the human lung from single-cell RNA sequencing. Nature 587, 619-625 (2020).

16. Lukassen, S. et al. SARS -CoV-2 receptor ACE 2 and TMPRSS 2 are primarily expressed in bronchial transient secretory cells. EMBO J. 39, e10511 (2020).

17. Ziegler, C. G. K. et al. SARS-CoV-2 receptor ACE2 is an interferon-stimulated gene in human airway epithelial cells and is detected in specific cell subsets across tissues. Cell 181, 1016-1035.e19 (2020).

18. Zhou, Z. et al. Heightened innate immune responses in the respiratory tract of COVID-19 patients. Cell Host Microbe 27, 883-890.e2 (2020).

19. Lucas, C. et al. Longitudinal analyses reveal immunological misfiring in severe COVID-19. Nature 584, 463-469 (2020).

20. Onabajo, O. O. et al. Interferons and viruses induce a novel truncated ACE2 isoform and not the full-length SARS-CoV-2 receptor. Nat. Genet. 52 1283-1293 (2020).

21. Hadjadj, J. et al. Impaired type I interferon activity and inflammatory responses in severe COVID-19 patients. Sci. (80-). 369, 718-724 (2020).

22. Blanco-Melo, D. et al. Imbalanced host response to SARS-CoV-2 drives development of COVID-19. Cell 181, 1036-1045.e9 (2020).

23. Hung, I. F. N. et al. Triple combination of interferon beta- $1 \mathrm{~b}$, lopinavir-ritonavir, and ribavirin in the treatment of patients admitted to hospital with COVID-19: an open-label, randomised, phase 2 trial. Lancet 395, 1695-1704 (2020).

24. Guan, W. et al. Clinical characteristics of coronavirus disease 2019 in China. N. Engl. J. Med. 382, 1708-1720 (2020).

25. Giacomelli, A. et al. Self-reported olfactory and taste disorders in SARS-CoV-2 patients: a cross-sectional study. Clin. Infect. Dis. 71, 889-890 (2020).

26. Huang, $C$. et al. Clinical features of patients infected with 2019 novel coronavirus in Wuhan, China. Lancet 39, 497-506 (2020).

27. Qi, F., Qian, S., Zhang, S. \& Zhang, Z. Single cell RNA sequencing of 13 human tissues identify cell types and receptors of human coronaviruses. Biochem. Biophys. Res. Commun. 526, 135-140 (2020).

28. Zou, $X$. et al. Single-cell RNA-seq data analysis on the receptor ACE2 expression reveals the potential risk of different human organs vulnerable to 2019-nCoV infection. Front. Med. 14, 185-192 (2020).

29. Sungnak, W. et al. SARS-CoV-2 entry factors are highly expressed in nasal epithelial cells together with innate immune genes. Nat. Med. 26, 681-687 (2020).

30. Zang, R. et al. TMPRSS2 and TMPRSS4 promote SARS-CoV-2 infection of human small intestinal enterocytes. Sci. Immunol. 5, eabc3582 (2020).

31. Ma, D. et al. Expression of SARS-CoV-2 receptor ACE2 and TMPRSS2 in human primary conjunctival and pterygium cell lines and in mouse cornea. Eye 34, 1212-1219 (2020).

32. Chen, L. et al. Ocular manifestations of a hospitalised patient with confirmed 2019 novel coronavirus disease. Br. J. Ophthalmol. 104, 748-751 (2020).

33. Chen, L., Li, X., Chen, M., Feng, Y. \& Xiong, C. The ACE2 expression in human heart indicates new potential mechanism of heart injury among patients infected with SARS-CoV-2. Cardiovasc. Res. 116, 1097-1100 (2020).

34. Yang, J. et al. Pathological Ace2-to-Ace enzyme switch in the stressed heart is transcriptionally controlled by the endothelial Brg1-FoxM1 complex. Proc. Natl Acad. Sci. USA 113, E5628-E5635 (2016).

35. Grobe, J. L. et al. ACE2 overexpression inhibits hypoxia-induced collagen production by cardiac fibroblasts. Clin. Sci. 113, 357-364 (2007).

36. Puelles, V. G. et al. Multiorgan and renal tropism of SARS-CoV-2. N. Engl. J. Med. 383, 590-592 (2020). 
37. Varga, Z. et al. Endothelial cell infection and endothelitis in COVID-19. Lancet 395, 1417-1418 (2020).

38. Pujadas, E. et al. SARS-CoV-2 viral load predicts COVID-19 mortality. Lancet Respir. Med. 8, e70 (2020).

39. Fajnzylber, J. et al. SARS-CoV-2 viral load is associated with increased disease severity and mortality. Nat. Commun. 11, 1-9 (2020).

40. Argyropoulos, K. V. et al. Association of initial viral load in severe acute respiratory syndrome coronavirus 2 (SARS-CoV-2) patients with outcome and symptoms. Am. J. Pathol. 190, 1881-1887 (2020).

41. Dorward, D. A. et al. Tissue-specific Immunopathology in Fatal COVID-19. Am. J. Respir. Crit. Care Med. https://doi.org/10.1164/rccm.202008-3265oc (2020).

42. Jin, J. M. et al. Gender differences in patients with COVID-19: focus on severity and mortality. Front. Public Heal. 8, 152 (2020).

43. Muus, C. et al. Integrated analyses of single-cell atlases reveal age, gender, and smoking status associations with cell type-specific expression of mediators of SARS-CoV-2 viral entry and highlights inflammatory programs in putative target cells. Preprint at https://doi.org/10.1101/2020.04.19.049254 (2020)

44. Chen, Y., Shan, K. \& Qian, W. Asians do not exhibit elevated expression or unique genetic polymorphisms for ACE2, the cell-entry receptor of SARSCoV-2. Preprint at https:/www.preprints.org/manuscript/202002.0258/v2 (2020).

45. Li, G. et al. Assessing ACE2 expression patterns in lung tissues in the pathogenesis of COVID-19. J. Autoimmun. 112, 102463 (2020).

46. Tukiainen, $\mathrm{T}$. et al. Landscape of $\mathrm{X}$ chromosome inactivation across human tissues. Nature 550, 244-248 (2017).

47. Liu, J. et al. Sex differences in renal angiotensin converting enzyme 2 (ACE2) activity are 17/-oestradiol-dependent and sex chromosome-independent. Biol. Sex. Differ. 1, 6 (2010)

48. Bukowska, A. et al. Protective regulation of the ACE2/ACE gene expression by estrogen in human atrial tissue from elderly men. Exp. Biol. Med. 242 1412-1423 (2017).

49. Schreiber, J., Bilmes, J. \& Noble, W. S. Completing the ENCODE3 compendium yields accurate imputations across a variety of assays and human biosamples. Genome Biol. 21, 82 (2020).

50. Biddie, S. C. et al. Transcription factor AP1 potentiates chromatin accessibility and glucocorticoid receptor binding. Mol. Cell https://doi.org/10.1016/j. molcel.2011.06.016 (2011).

51. Lin, B. et al. Prostate-localized and androgen-regulated expression of the membrane- bound serine protease TMPRSS2. Cancer Res. 59, 4180-4184 (1999).

52. Tomlins, S. A. et al. Recurrent fusion of TMPRSS2 and ETS transcription factor genes in prostate cancer. Sci. (80-). 310, 644-648 (2005).

53. Lamy, P. J., Rébillard, X., Vacherot, F. \& de la Taille, A. Androgenic hormones and the excess male mortality observed in COVID-19 patients: new convergent data. World J. Urol. https://doi.org/10.1007/s00345-020-03284-y (2020).

54. Wambier, C. G. et al. Androgenetic alopecia present in the majority of patients hospitalized with COVID-19: The "Gabrin sign". J. Am. Acad. Dermatol. 83, 680-682 (2020)

55. Baratchian, M. et al. Sex, androgens and regulation of pulmonary $A R$, TMPRSS2 and ACE2. bioRxiv Prepr. Sen. Biol. https://doi.org/10.1101/ 2020.04.21.051201 (2020).

56. Baena, E. et al. ETV1 directs androgen metabolism and confers aggressive prostate cancer in targeted mice and patients. Genes Dev. 27, 683-698 (2013).

57. Parascandola, M. \& Xiao, L. Tobacco and the lung cancer epidemic in China. Transl. Lung Cancer Res. 8, S21-S30 (2019).

58. Breidenbach, J. D. et al. Impact of comorbidities on SARS-CoV-2 viral entryrelated genes. J. Pers. Med. 10, 1-11 (2020).

59. Leung, J. M. et al. ACE-2 expression in the small airway epithelia of smokers and COPD patients: implications for COVID-19. Eur. Respir. J. 55, 2000688 (2020).

60. Smith, J. C. et al. Cigarette smoke exposure and inflammatory signaling increase the expression of the SARS-CoV-2 receptor ACE2 in the respiratory tract. Dev. Cell 53, 514-529.e3 (2020).

61. Chen, J. et al. Individual variation of the SARS-CoV-2 receptor ACE2 gene expression and regulation. Aging Cell 19, e13168 (2020).

62. To, K. K. W. et al. Temporal profiles of viral load in posterior oropharyngeal saliva samples and serum antibody responses during infection by SARS-CoV2: an observational cohort study. Lancet Infect. Dis. 20, 565-574 (2020).
63. Schuler, B. A. et al. Age-determined expression of priming protease TMPRSS2 and localization of SARS-CoV-2 in lung epithelium. J. Clin. Invest. 131, e140766 (2020).

64. Wang, J. et al. Respiratory influenza virus infection induces intestinal immune injury via microbiotamediated Th17 cell-dependent inflammation. J. Exp. Med. 211, 2397-2410 (2014).

65. Bunyavanich, S., Do, A. \& Vicencio, A. Nasal gene expression of angiotensinconverting enzyme 2 in children and adults. JAMA 363, 2427-2429 (2020).

66. Dong, Y. et al. Epidemiology of COVID-19 among children in China. Pediatrics 145, 20200702 (2020).

67. Lee, N. et al. IFTMM3, TLR3, and CD55 Gene SNPs and cumulative genetic risks for severe outcomes in chinese patients With H7N9/H1N1pdm09 influenza. J. Infect. Dis. 216, 97-104 (2017).

68. Tu, X. et al. Functional polymorphisms of the CCL2 and MBL genes cumulatively increase susceptibility to severe acute respiratory syndrome coronavirus infection. J. Infect. 71, 101-109 (2015).

69. Karczewski, $\mathrm{K}$. J. et al. The mutational constraint spectrum quantified from variation in 141,456 humans. Nature $\mathbf{5 8 1}, 434-443$ (2020).

70. Asselta, R., Paraboshi, E., Montovani, A. \& Duga, S. ACE2 and TMPRSS2 variants and expression as candidates to sex and country differences in COVID-19 severity in Italy. Aging (Albany NY). 12, 10087-10098 (2020).

71. Cheng, Z. et al. Identification of TMPRSS2 as a susceptibility gene for severe 2009 pandemic $A(H 1 N 1)$ influenza and $A(H 7 N 9)$ influenza. J. Infect. Dis. 212, 1214-1221 (2015).

72. Zhang, Q. et al. Inborn errors of type I IFN immunity in patients with lifethreatening COVID-19. Science 370, eabd4570 (2020).

73. Ishida, H. et al. Cryo-EM structures of Toll-like receptors in complex with UNC93B1. Nat. Struct. Mol. Biol. https://doi.org/10.1038/s41594-020-00542-w (2021).

74. Kreins, A. Y. et al. Human TYK2 deficiency: mycobacterial and viral infections without hyper-lgE syndrome. J. Exp. Med. 212, 1641-1662 (2015).

75. Combes, A. J. et al. Global absence and targeting of protective immune states in severe COVID-19. Nature https://doi.org/10.1038/s41586-021-032347 (2021).

76. WHO Solidarity Trial Consortium, Pan, H., Peto, R., AM, H.-R. \& Preziosi, M. Repurposed antiviral drugs for covid-19-interim WHO solidarity trial results. N. Engl. J. Med. 384, 497-511 (2020).

77. Gadotti, A. C. et al. IFN- $\gamma$ is an independent risk factor associated with mortality in patients with moderate and severe COVID-19 infection. Virus Res. 289, 198171 (2020).

78. Hurst, S. M. et al. IL-6 and its soluble receptor orchestrate a temporal switch in the pattern of leukocyte recruitment seen during acute inflammation. Immunity 14, 705-714 (2001).

79. Bovijn, J., Lindgren, C. M. \& Holmes, M. V. Genetic variants mimicking therapeutic inhibition of IL-6 receptor signaling and risk of COVID-19. Lancet Rheumatol. 2, e658-e659 (2020).

80. REMAP-CAP Investigators et al. Interleukin-6 receptor antagonists in critically III patients with covid-19-. N. Engl. J. Med. https://doi.org/10.1056/ NEJMoa2100433 (2021).

81. Salama, C. et al. Tocilizumab in patients hospitalized with covid-19 pneumonia. N. Engl. J. Med. https://doi.org/10.1056/nejmoa2030340 (2020).

82. Ellinghaus, D. et al. Genomewide Association Study of Severe Covid-19 with Respiratory Failure. N. Engl. J. Med. 383, 1522-1534 (2020).

83. Vuille-Dit-Bille, R. N. et al. Human intestine luminal ACE2 and amino acid transporter expression increased by ACE-inhibitors. Amino Acids 47, 693-70 (2015).

84. Wein, A. N. et al. CXCR6 regulates localization of tissue-resident memory CD8 T cells to the airways. J. Exp. Med. 216, 2748-2762 (2019).

85. Thavarajah, T. et al. The plasma peptides of sepsis. Clin. Proteom. 17, 26 (2020).

86. Zhao, J. et al. Relationship between the ABO Blood Group and the COVID-19 susceptibility. Clin. Infect. Dis. ciaa1150 (2020).

87. Choi, U. Y., Kang, J. S., Hwang, Y. S. \& Kim, Y. J. Oligoadenylate synthase-like (OASL) proteins: dual functions and associations with diseases. Exp. Mol. Med. 47, e144 (2015).

88. Shepardson, K. M. et al. IFNAR2 is required for anti-influenza immunity and alters susceptibility to post-influenza bacterial superinfections. Front. Immunol. 9, 2589 (2018).

89. Fingerlin, T. E. et al. Genome-wide association study identifies multiple susceptibility loci for pulmonary fibrosis. Nat. Genet. 45, 613-620 (2013).

90. Maurano, M. T. et al. Systematic localization of common disease-associated variation in regulatory DNA. Science 337, 1190-1195 (2012). 
91. Thurman, R. E. et al. The accessible chromatin landscape of the human genome. Nature 489, 75-82 (2012).

92. Kasowski, M. et al. Variation in transcription factor binding among humans. Science 328, 232-235 (2010).

93. Dixon, J. R. et al. Topological domains in mammalian genomes identified by analysis of chromatin interactions. Nature https://doi.org/10.1038/ nature11082 (2012).

94. Schmitt, A. D. et al. A compendium of chromatin contact maps reveals spatially active regions in the human genome. Cell Rep. 17, 2042-2059 (2016).

95. Rao, S. S. P. et al. A 3D map of the human genome at kilobase resolution reveals principles of chromatin looping. Cell 159, 1665-1680 (2014).

96. Szabo, Q., Bantignies, F. \& Cavalli, G. Principles of genome folding into topologically associating domains. Sci. Adv. 5, eaaw1668 (2019).

97. Kumasaka, N., Knights, A. J. \& Gaffney, D. J. High-resolution genetic mapping of putative causal interactions between regions of open chromatin. Nat. Genet. 51, 128-137 (2019).

98. Chen, C. H. et al. Determinants of transcription factor regulatory range. Nat. Commun. 11, 2472 (2020).

99. Fadason, T. et al. A transcription regulatory network within the ACE2 locus may promote a pro-viral environment for SARS-CoV-2 by modulating expression of host factors. Preprint at https://doi.org/10.1101/ 2020.04.14.042002 (2020).
100. Wang, A. et al. Single cell multiomic profiling of human lung reveals cell type-specific and age-dynamic control of SARS-CoV2 host genes. Elife $\mathbf{9}$, e62522 (2020).

101. Zhang, X. et al. Viral and host factors related to the clinical outcome of COVID-19. Nature 583, 437-440 (2020).

102. Imai, Y. et al. Angiotensin-converting enzyme 2 protects from severe acute lung failure. Nature 436, 112-116 (2005).

103. RECOVERY Collaborative Group et al. Dexamethasone in hospitalized patients with covid-19-preliminary report. N. Engl. J. Med. 384, 693-704 (2020).

104. Angus, D. C. et al. Effect of hydrocortisone on mortality and organ support in patients with severe COVID-19: The REMAP-CAP COVID-19 corticosteroid domain randomized clinical. JAMA 324, 1317-1329 (2020).

105. Li, B. et al. Genome-wide CRISPR screen identifies host dependency factors for influenza A virus infection. Nat. Commun. 11, 164 (2020).

106. Melnikov, A. et al. Systematic dissection and optimization of inducible enhancers in human cells using a massively parallel reporter assay. Nat. Biotechnol. 30, 271-277 (2012).

107. GTEx Consortium. et al. The Genotype-Tissue Expression (GTEx) pilot analysis: multitissue gene regulation in humans. Science 348, 648-660 (2015).

108. Leung, D. et al. Integrative analysis of haplotype-resolved epigenomes across human tissues. Nature 518, 350-354 (2015).

109. Kerpedjiev, P. et al. HiGlass: web-based visual exploration and analysis of genome interaction maps. Genome Biol. 19, 125 (2018). 